\title{
High-energy elastic diffractive scattering of nucleons in the framework of the two-Reggeon eikonal approximation (from U-70 to LHC)
}

\author{
A. A. Godizov ${ }^{\text {a }}$ \\ A.A. Logunov Institute for High Energy Physics, NRC "Kurchatov Institute”, 142281 Protvino, Russia
}

Received: 28 October 2021 / Accepted: 10 January 2022 / Published online: 20 January 2022

(C) The Author(s) 2022

\begin{abstract}
Elastic diffractive scattering of nucleons is described in terms of Regge-eikonal approach. It is demonstrated that, in a wide kinematic region (starting from the U-70 energies), the eikonal of proton-proton scattering can be approximated by the sum of two Reggeon exchange terms (namely, the so-called soft Pomeron and $f$-Reggeon contributions). The range of applicability of the considered approximation is determined. The predictive value of the proposed phenomenological scheme is verified.
\end{abstract}

\section{Introduction}

Diffraction phenomena in high-energy hadron physics are related to comparatively large transverse distances between interacting hadrons, from 0.1 to $10 \mathrm{fm}$. This fact leads to a specific situation when the fundamental theory of strong interaction, quantum chromodynamics (QCD), or, more exactly, perturbative QCD, does not help us to describe diffractive scattering of hadrons and to make reliable predictions for the observables of the corresponding reactions. Therefore, the most of modern models of hadron diffraction are formulated rather in terms of hadrodynamics itself than in terms of interacting quarks and gluons. As a consequence, these models reveal a very low predictive value (for detailed discussion, see a minireview in [1]).

Nonetheless, hadron diffraction processes constitute an important sector of high-energy physics, owing, first of all, to the high fraction of diffraction events in the total number of events at high-energy hadron colliders (not less than $30 \%$ at the LHC energies). Hence, approaches are needed which, on the one hand, allow to make reliable predictions (at least, qualitative) for hadron diffraction observables, and, on the

a e-mail: anton.godizov@gmail.com (corresponding author) other hand, admit connection with QCD in the future, in the case of essential progress in nonperturbative QCD methods.

The aim of this paper is to demonstrate that a simple approximation can be constructed in the framework of the general Regge-eikonal approach, which allows to describe the high-energy evolution of the diffraction pattern of nucleon-nucleon elastic diffractive scattering (EDS) within a very large kinematic range.

The Regge-eikonal formalism itself is expounded in detail in $[2,3]$. Therefore, in what follows, we restrict ourselves just by a concise review of its main points. Next, using some QCD-based arguments, we formulate the two-Reggeon approximation and apply it to available data on high-energy EDS of nucleons, for determination of the proposed model applicability range and demonstration of its predictive value. Then, we discuss the issues and compare the considered phenomenological scheme with other models of high-energy EDS which exploit the notion of Reggeon.

\section{Basics of Regge-eikonal approach}

\subsection{Eikonal representation and the Van Hove hypothesis}

Regge-eikonal approximation is founded on the eikonal representation of the high-energy elastic scattering amplitude [2]:

$$
\begin{aligned}
& T_{N}(s, t)=4 \pi s \int_{0}^{\infty} d\left(b^{2}\right) J_{0}(b \sqrt{-t}) \frac{e^{2 i \delta_{N}(s, b)}-1}{2 i}, \\
& \delta_{N}(s, b)=\frac{1}{16 \pi s} \int_{0}^{\infty} d(-t) J_{0}(b \sqrt{-t}) \Omega_{N}(s, t),
\end{aligned}
$$

where $s$ and $t$ are the Mandelstam variables, $b$ is the impact parameter, $J_{0}(x)$ is the Bessel function, $T_{N}$ is the full amplitude related to strong interaction, the eikonal $\delta_{N}$ is the two- 
particle-irreducible part of $T_{N}$ in the coordinate representation, and the function $\Omega_{N}$ is the eikonal in the transferred momentum representation.

Such a form of the full amplitude resembles the analogous formula for the amplitude of elastic scattering of nonrelativistic spinless particle in external spherically symmetric potential field obtained via exact solving of the corresponding Schrödinger equation. Then the eikonal is equal to the Born amplitude.

In high-energy hadron physics, the negligibility of multiparticle effects is not evident. Nonetheless, we have to presume their subdominance, as proposed in [4]. Otherwise, the representation (1) itself does not help us to solve the problem, since it is just reduced to the replacement of the unknown function of two variables, $T_{N}(s, t)$, by another unknown function, $\Omega_{N}(s, t)$, without any specification of the functional form of $\Omega_{N}(s, t)$.

Thus, the Van Hove hypothesis that, at high energies and low transferred momenta, the $s$-channel eikonal for elastic scattering of two spinless hadrons can be represented as

$\Omega_{N}(s, t)=\sum_{j} \sum_{m_{j}} \frac{\xi_{j} B^{\left(j, m_{j}\right)}(s, t)}{m_{j}^{2}-t}+\cdots$

is the first basic assumption necessary for construction of the Regge-eikonal approximation for the amplitude $T_{N}(s, t)$. Here $\xi_{j}$ is the signature factor, $B^{\left(j, m_{j}\right)}(s, t) \equiv$ $\beta^{\left(j, m_{j}\right)}(t) P_{j}\left(z_{t}\right), P_{j}(x)$ is the Legendre polynomial of degree $j, z_{t}$ is the cosine of the scattering angle in the $t$-channel, $m_{j}^{2} \equiv M_{j}^{2}-i M_{j} \Gamma_{j}$, where $j, M_{j}$, and $\Gamma_{j}$ are the resonance spin, mass, and full width of decay, correspondingly, and “..." denotes subdominant nonresonance contributions.

\subsection{Factorization of the residues}

However, the approximation (2) for the eikonal does not imply, by default, any factorization of the functions $\beta^{\left(j, m_{j}\right)}(t)$ into two factors each related directly to a certain one of the colliding spinless hadrons. Such a factorization emerges if we follow the formalism developed in [3], wherein the residue $B^{\left(j, m_{j}\right)}(s, t)$ is represented as the convolution of two currents dependent on the 4-momenta $p_{1}$ or $p_{2}$ of the interacting hadrons and on the transferred 4-momentum $\Delta\left(t \equiv \Delta^{2}\right)$ with some tensor related to the exchanged virtual resonance and dependent on $\Delta$ only:

$$
\begin{aligned}
B^{\left(j, m_{j}\right)}(s, t)= & J_{\mu_{1} \ldots \mu_{j}}^{\left(h_{1}, m_{j}\right)}\left(p_{1}, \Delta\right) D_{\left(m_{j}\right)}^{\mu_{1} \ldots \mu_{j} ; v_{1} \ldots v_{j}}(\Delta) \\
& \times J_{v_{1} \ldots v_{j}}^{\left(h_{2}, m_{j}\right)}\left(p_{2},-\Delta\right) .
\end{aligned}
$$

Here $J_{\mu_{1} \ldots \mu_{j}}^{\left(h_{1,2}, m_{j}\right)}\left(p_{1,2}, \Delta\right)$ are the currents of the colliding particles, the indices $h_{1,2}$ denote the sort of the hadron, and the index $m_{j}$ indicates that the properties of $J$ and $D$ depend on the physical structure of the exchanged virtual resonance state (below it will be omitted).

If we represent the tensor structure of $J_{\mu_{1} \ldots \mu_{j}}^{(h)}(p, \Delta)$ in the most general form,

$J_{\mu_{1} \ldots \mu_{j}}^{(h)}(p, \Delta)=g_{j}^{(h)}(t) p_{\mu_{1}} \ldots p_{\mu_{j}} s_{0}^{-j / 2}+\cdots$

(where $s_{0}=1 \mathrm{GeV}^{2}$ is the unit of measurement, while "..." denotes the combined contribution of the tensor substructures different from $p_{\mu_{1}} \ldots p_{\mu_{j}}$ ) and convolve both the currents with

$$
\begin{aligned}
& D^{\mu_{1} \ldots \mu_{j} ; v_{1} \ldots v_{j}}(\Delta)=\frac{f_{j}(t)}{j !}\left[g^{\mu_{1} v_{1}} \ldots g^{\mu_{j} v_{j}}\right. \\
& \left.+ \text { all the permutations of } v_{k}\right]+\cdots \text {, }
\end{aligned}
$$

then we obtain

$$
\begin{aligned}
B^{(j)}(s, t)= & g_{j}^{\left(h_{1}\right)}(t) g_{j}^{\left(h_{2}\right)}(t) f_{j}(t) \\
& \times\left(\frac{\left(p_{1} p_{2}\right)}{s_{0}}\right)^{j}\left[1+O\left(\left(p_{1} p_{2}\right)^{-1}\right)\right] .
\end{aligned}
$$

Hence, in the kinematic region $\left(p_{1}+p_{2}\right)^{2} \equiv s \gg$ $\left\{p_{1,2}^{2}, s_{0},|t|\right\}$, we come to the following high-energy approximation for the residue $B$ :

$B^{(j)}(s, t) \approx \tilde{g}_{j}^{\left(h_{1}\right)}(t) \tilde{g}_{j}^{\left(h_{2}\right)}(t)\left(\frac{s}{2 s_{0}}\right)^{j}$

where the factors $\tilde{g}_{j}^{\left(h_{1,2}\right)}(t) \equiv g_{j}^{\left(h_{1,2}\right)}(t) f_{j}^{1 / 2}(t)$ could be interpreted as the effective couplings of the exchanged virtual resonance to the interacting hadrons.

\subsection{Helicity currents of protons}

In the case of the high-energy scattering of protons, we should not a priori ignore the spin-flip effects, but it is possible to show that they are subdominant at small enough values of the transferred momentum.

Let $u^{(1)}(p)\left(u^{(2)}(p)\right)$ be the Dirac spinor related to the proton state with 4-momentum $p$ and positive (negative) helicity, and let the normalization of these spinors be fixed by the relation

$\sum_{i} u_{\alpha}^{(i)}(p) \bar{u}_{\beta}^{(i)}(p)=\left(\hat{p}+m_{p} I\right)_{\alpha \beta}$,

where $\alpha$ and $\beta$ are the spinor indices, $m_{p}$ is the proton mass, $I$ is the unity matrix, $\bar{u}^{(i)} \equiv u^{(i)+} \gamma^{0}, \hat{p} \equiv p_{\mu} \gamma^{\mu}$, and $\gamma^{\mu}$ 
are the Dirac matrices. Then, the most general form for an arbitrary helicity current of proton is

$$
\begin{aligned}
& J_{\left(i i^{\prime}\right)}^{\mu_{1} \ldots \mu_{j}}(p, \Delta)=\bar{u}_{\alpha}^{\left(i^{\prime}\right)}(p+\Delta)\left(g_{j}^{(0)}(t) I_{\alpha \beta} p^{\mu_{1}} \ldots p^{\mu_{j}} s_{0}^{-j / 2}\right. \\
& \quad+\frac{g_{j}^{(1)}(t)}{j}\left[\gamma_{\alpha \beta}^{\mu_{1}} p^{\mu_{2}} \ldots p^{\mu_{j}}\right. \\
& \left.+ \text { permutations between } \mu_{1} \text { and } \mu_{k}\right] s_{0}^{-(j-1) / 2} \\
& \quad+\cdots) u_{\beta}^{(i)}(p) .
\end{aligned}
$$

Taking into account that $s \gg\left\{m_{p}^{2}, s_{0},|t|\right\}$ and summing over the spinor indices, we obtain, in the leading approximation, that

$$
\begin{aligned}
& J_{21}^{\mu_{1} \ldots \mu_{j}}(p, \Delta)=-J_{12}^{\mu_{1} \ldots \mu_{j}}(p, \Delta) \\
& \quad=\sqrt{-t} g_{j}^{(0)}(t) p^{\mu_{1}} \ldots p^{\mu_{j}} s_{0}^{-j / 2}+\cdots
\end{aligned}
$$

and

$$
\begin{aligned}
& J_{11}^{\mu_{1} \ldots \mu_{j}}(p, \Delta)=J_{22}^{\mu_{1} \ldots \mu_{j}}(p, \Delta) \\
& \quad=\left[2 m_{p} g_{j}^{(0)}(t)+2 \sqrt{s_{0}} g_{j}^{(1)}(t)\right] p^{\mu_{1}} \ldots p^{\mu_{j}} s_{0}^{-j / 2}+\cdots
\end{aligned}
$$

Consequently, we can ignore the spin effects in the region of small enough values of the transferred momentum, wherein the following inequality is valid:

$$
\sqrt{-t}\left|g_{j}^{(0)}(t)\right| \ll\left|2 m_{p} g_{j}^{(0)}(t)+2 \sqrt{s_{0}} g_{j}^{(1)}(t)\right| \equiv\left|g_{j}^{(p)}(t)\right| .
$$

The measurements performed by STAR Collaboration [5] demonstrate that, in the range $\sqrt{-t}<0.2 \mathrm{GeV}$, the spin-flip events take place due to electromagnetic interaction only. In their turn, available data on the proton-proton EDS at lower energies [6-8] point to the fact that, in the interval $0.5 \mathrm{GeV}$ $<\sqrt{-t}<1.5 \mathrm{GeV}$, the spin-flip contributions affect the differential cross-section very slightly, and, thus, they could be considered negligible, in the leading approximation (detailed discussion of this matter can be found in a recent paper [9]). Therefore, in further, we assume their subdominance in the whole kinematic range relevant for the high-energy EDS of nucleons. In this case, we may treat nucleons as spinless particles, and direct exploitation of the Regge-eikonal representation (1) of the scattering amplitude is justified.

\subsection{Reggeization}

Next, let us consider the combined contribution into the elastic scattering eikonal by a family of $t$-channel resonance terms, and let every term in this family to be related to some meson or glueball state with nonzero even value of the spin $j(j=2,4,6, \ldots)$. As it has been discussed above, in the kinematic sector $s \gg\left\{p_{1,2}^{2}, s_{0},|t|\right\}$ of the $s$-channel physical region, this contribution can be approximated by the expression

$\Omega_{+}(s, t)=\sum_{j=2}^{\infty} \frac{1+e^{-i \pi j}}{m_{j}^{2}-t}\left(\frac{s}{2 s_{0}}\right)^{j} \tilde{g}_{j}^{\left(h_{1}\right)}(t) \tilde{g}_{j}^{\left(h_{2}\right)}(t)$.

If $\tilde{g}_{j}^{(h)}(t)$ and $m_{j}^{2}$ are the values at positive even $j$ of some analytic functions which are holomorphic in the region $\operatorname{Re} j>0$ and behave as $O\left(e^{k|j|}\right), k<\pi$, at $j \rightarrow \infty$, then, under the Carlson theorem, the unilocal analytic continuation of (13) is possible into the region of complex $j$ (the Regge hypothesis [2]). We denote these functions by $\tilde{g}^{(h)}(t, j)$ and $m^{2}(j)$, respectively. Via the Sommerfeld-Watson transform $[2,10,11]$, we can replace the sum over $j$ in (13) by the integral over the contour $C$ encircling the real positive half-axis in the complex $j$-plane, including the point $j=2$ (but not including the point $j=0$ ), in such a way that the half-axis is on the right:

$$
\begin{aligned}
\Omega_{+}(s, t)= & -\frac{1}{2 i} \oint_{C} \frac{d j}{\sin (\pi j)} \frac{1+e^{-i \pi j}}{m^{2}(j)-t} \\
& \times\left(\frac{s}{2 s_{0}}\right)^{j} \tilde{g}^{\left(h_{1}\right)}(t, j) \tilde{g}^{\left(h_{2}\right)}(t, j) .
\end{aligned}
$$

According to our assumptions, the only sources of the integrand singularities in the region $\operatorname{Re} j>0$ are the zeros of the functions $\sin (\pi j)$ and $m^{2}(j)-t$. Hence, deforming the contour $C$ to the axis $\operatorname{Re} j=\epsilon \rightarrow+0$ (the asymptotic behavior of the functions $\tilde{g}^{(h)}(t, j)$ at $j \rightarrow \infty$ in the region $\operatorname{Re} j>0$ and $t \leq 0$ should allow such a deformation), we obtain

$$
\begin{aligned}
& \Omega_{+}(s, t)=-\frac{1}{2 i} \int_{\epsilon-i \infty}^{\epsilon+i \infty} \frac{d j}{\sin (\pi j)} \\
& \quad \times \frac{1+e^{-i \pi j}}{m^{2}(j)-t}\left(\frac{s}{2 s_{0}}\right)^{j} \tilde{g}^{\left(h_{1}\right)}(t, j) \tilde{g}^{\left(h_{2}\right)}(t, j) \\
& -\frac{1+e^{-i \pi \alpha(t)}}{\sin (\pi \alpha(t))} \pi \alpha^{\prime}(t)\left(\frac{s}{2 s_{0}}\right)^{\alpha(t)} g^{\left(h_{1}\right)}(t) g^{\left(h_{2}\right)}(t) .
\end{aligned}
$$

Here $g^{(h)}(t) \equiv \tilde{g}^{(h)}(t, \alpha(t))$, while the analytic function $\alpha(t)$ is the root of the equation $m^{2}(j)-t=0$. We assume, for simplicity, that, at $t \leq 0$, this equation has a unique $t$ dependent root in the region $\operatorname{Re} j>0$, wherein the functions $\tilde{g}^{(h)}(t, j)$ and $m^{2}(j)$ are holomorphic. Thus, $\alpha(t)$ is related to a moving pole in the complex $j$-plane. Such poles are called Regge poles, and the corresponding functions $\alpha(t)$ are called Regge trajectories.

As far as $\operatorname{Re} \alpha(t)>0$, the background integral is negligible at high enough values of $s$, and, finally, we come to the following high-energy approximation for the contribution of any even-spin Reggeon to the eikonal: 
$\Omega_{+}(s, t) \approx \xi_{+}(\alpha(t)) \alpha^{\prime}(t)\left(\frac{s}{2 s_{0}}\right)^{\alpha(t)} g^{\left(h_{1}\right)}(t) g^{\left(h_{2}\right)}(t)$,

where $\xi_{+}(\alpha(t))$ denotes the so-called Reggeon signature factor for the even-spin Reggeon associated with the Regge trajectory $\alpha(t)$ :

$\xi_{+}(\alpha(t))=\pi\left(i+\tan \frac{\pi(\alpha(t)-1)}{2}\right)$.

In the case of an odd-spin Reggeon, the corresponding factor would be [2]

$\xi_{-}(\alpha(t))=\pi\left(i-\cot \frac{\pi(\alpha(t)-1)}{2}\right)$.

Formula (16) together with the eikonal representation (1) of the elastic scattering amplitude, where the eikonal is just the sum of a few Reggeon contributions, is the essence of Regge-eikonal approach.

\section{Two-Reggeon eikonal model}

\subsection{Specific structure of the eikonal}

The main problem emerging in the framework of Reggeeikonal approach and, in general, in Regge theory is the evident functional indeterminacy. In particular, describing the high-energy EDS of nucleons, we have to introduce two unknown functions for every Reggeon term, namely, the Regge trajectory and the corresponding Reggeon coupling to nucleon. The energy evolution of the integrated cross-section of proton-proton elastic scattering [12,13] (see Fig. 1) points to the existence of two different sorts of Reggeons:

- supercritical Reggeons with the Regge trajectory intercept higher than the unity which are responsible for the cross-section growth at high energies;

- secondary Reggeons responsible for the cross-section decrease at low energies.

In literature, secondary Reggeons are usually associated with the families of the Quark Model low-mass vector and tensor mesons ( $\omega, f, \rho, a, \phi$, etc.), while the even-spin (oddspin) supercritical Reggeons called Pomerons (Odderons) are assumed to be related to glueballs.

The most important question is how many Reggeons contribute crucially into the eikonal of nucleon-nucleon EDS at available energies. Different models give different answers. However, the fundamental quantum field model of strong interaction helps to clarify the general structure of the secondary Reggeon part of the nucleon-nucleon EDS eikonal.

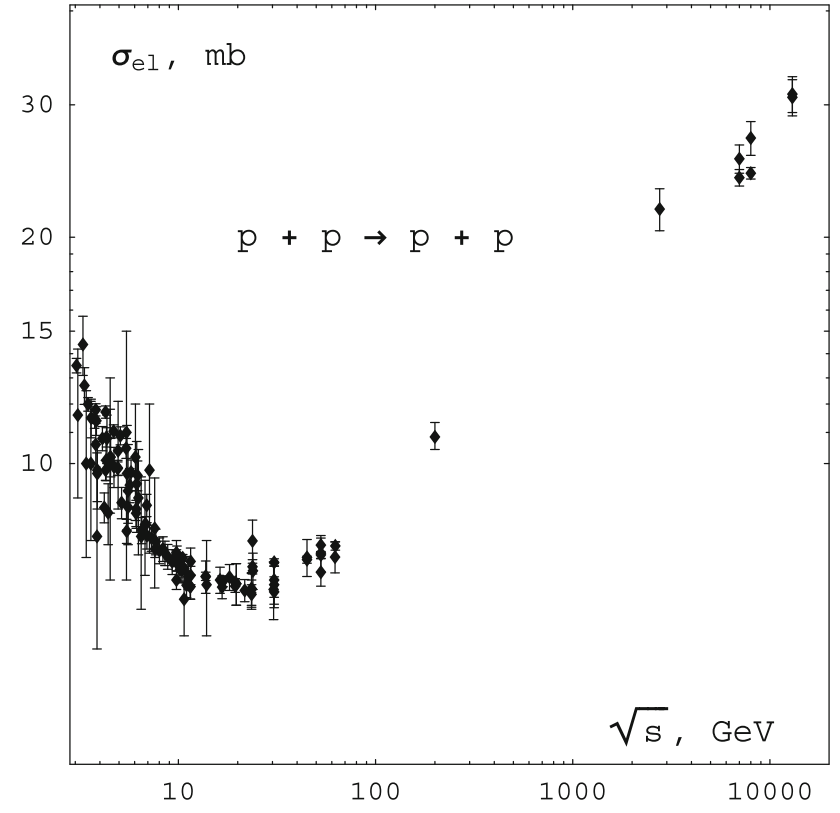

Fig. 1 The energy evolution of the $p p$ EDS integrated cross-section $[12,13]$

Namely, two types of the secondary Reggeon exchange contributions emerge in the QCD dynamics of nucleons:

- the contributions of type 1 related to the exchange by valence quarks between nucleons (the left picture in Fig. 2);

- the contributions of type 2 related to those QCD terms wherein the exchange by valence quarks does not take place (the right picture in Fig. 2).

Moreover, it was explicitly shown in the framework of the so-called dual approximation of QCD [14] that the contributions of type 1 to nucleon-nucleon interaction are purely real. Therefore, if we represent the secondary Reggeon part of the nucleon-nucleon EDS eikonal in terms of $C$-even and $C$-odd Reggeon exchanges, then we come to the following structure of the nucleon-nucleon and nucleon-antinucleon diffractive interaction:

$$
\begin{aligned}
\Omega_{N}^{(s e c)}(s, t) \approx & \Omega_{\mathrm{FR}}(s, t)+\Omega_{f}(s, t) \mp \Omega_{\omega}(s, t) \\
& +\Omega_{a}(s, t) \mp \Omega_{\rho}(s, t)
\end{aligned}
$$

where $\Omega_{\mathrm{FR}}(s, t)$ is the $f$-Reggeon (FR) contribution of type 2 related to the QCD terms without exchange by valence quarks between the nucleons, $\Omega_{f}(s, t)$ is the contribution of type 1 of the same Reggeon, the last three terms are related to the secondary Reggeons $\omega, a$, and $\rho$, and the sign "-_" ("+") at the $C$-odd terms corresponds to the nucleon-nucleon (nucleon-antinucleon) interaction.

If the imaginary parts of $\Omega_{f}(s, t)$ and $\Omega_{\omega}(s, t)$ and, also, of $\Omega_{a}(s, t)$ and $\Omega_{\rho}(s, t)$ coincide (the so-called exchange 
Fig. 2 Two types of the QCD contributions to

nucleon-nucleon EDS wherein the meson resonance exchange terms emerge
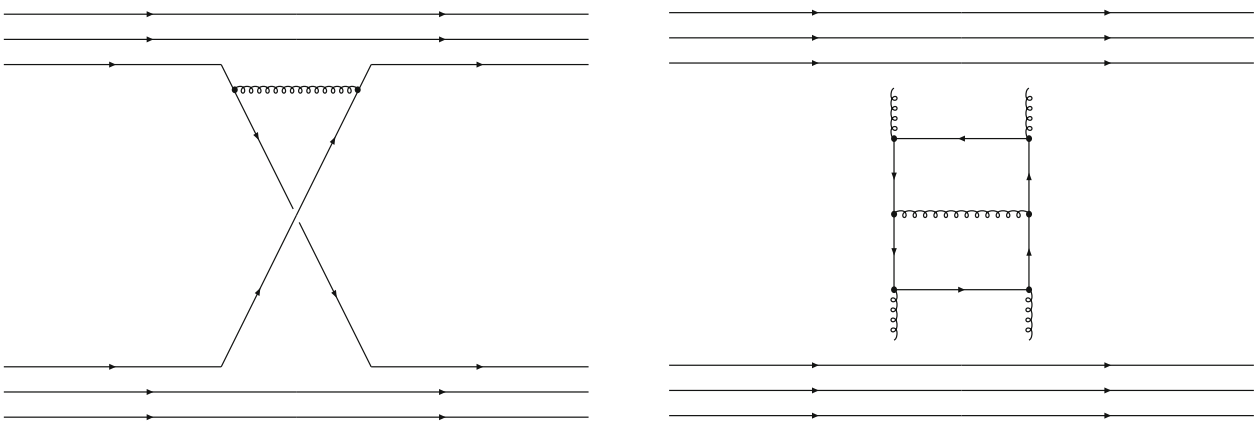

degeneracy phenomenon), then they annihilate each other in the $p p$ EDS regime, in accordance with the physical pattern emerging in the framework of the dual approximation of QCD. Therefore, in the case of the $p p$ EDS, the contributions of type 1 can be neglected, in the leading approximation, and, thus, only the FR exchange term of type 2 is crucial for the p $p$ EDS. ${ }^{1}$

Regarding the supercritical Reggeons, the situation is much more indefinite. No argument exists which limits $a$ priori the number of crucial Pomerons or Odderons. As a consequence, their amount varies from model to model. In this paper, we restrict ourselves by the simplest variant, wherein just one supercritical Reggeon is assumed to give the leading contribution within the relevant kinematic range, namely, the so-called soft Pomeron (SP). Thus, the considered approximation is reduced to the representation of the $p p$ EDS eikonal as the sum of two Regge-pole terms related to the SP and the FR:

$\Omega_{N}(s, t) \approx \Omega_{\mathrm{SP}}(s, t)+\Omega_{\mathrm{FR}}(s, t)$.

Below, it will be demonstrated that this simplified phenomenological scheme (which ignores the secondary Reggeon contributions of type 1) allows to reach a relatively good description of available data on the high-energy EDS of protons. As well, we will determine the kinematic range of applicability of approximation (20) and check its predictive value via application to available data at ultrahigh energies.

\subsection{General properties of the Regge trajectories and the Reggeon couplings to proton}

Hereby, in the framework of the proposed approximation, we have four unknown functions, namely, the Regge trajectories of the SP and the FR, and their couplings to proton: $\alpha_{\mathrm{SP}}(t)$, $\alpha_{\mathrm{FR}}(t), g_{\mathrm{SP}}^{(p)}(t)$, and $g_{\mathrm{FR}}^{(p)}(t)$. The main property of these functions is that they take real values at real values of $t$ below the

\footnotetext{
1 The only exclusion is a very small vicinity of the diffraction dip in the diffraction pattern of the $p p$ EDS at low enough values of the collision energy, wherein the combined secondary Reggeon contribution into the eikonal real part has an essential impact on the depth and shape of the diffraction dip.
}

two-pion threshold [2]:

$$
\begin{gathered}
\operatorname{Im} \alpha_{\mathrm{SP}}(t)=0, \operatorname{Im} \alpha_{\mathrm{FR}}(t)=0, \operatorname{Im} g_{\mathrm{SP}}^{(p)}(t)=0, \\
\operatorname{Im} g_{\mathrm{FR}}^{(p)}(t)=0 \quad\left(-\infty<t<4 m_{\pi^{0}}^{2}\right) .
\end{gathered}
$$

The absence of any points in $\alpha_{\mathrm{SP}}(t)$ and $\alpha_{\mathrm{FR}}(t)$ which are related to scalar mesons imposes the following restriction on their intercept values:

$\alpha_{\mathrm{SP}}(0)>0, \quad \alpha_{\mathrm{FR}}(0)>0$.

The unitarity condition for the elastic scattering amplitude in the impact parameter representation [2],

$0 \leq\left|T_{N}(s, b)\right|^{2} \leq \operatorname{Im} T_{N}(s, b) \leq 1$,

requires the eikonal imaginary part to be positive:

$\operatorname{Im} \delta_{N}(s, b) \geq 0$.

In its turn, the latter inequality takes place only if the functions $\alpha_{\mathrm{SP}}(t)$ and $\alpha_{\mathrm{FR}}(t)$ grow monotonically in the region of negative $t$ :

$\alpha_{\mathrm{SP}}^{\prime}(t)>0, \alpha_{\mathrm{FR}}^{\prime}(t)>0 \quad(-\infty<t \leq 0)$.

If the SP is related to some family of even-spin glueballs, and the quark-antiquark fraction in the SP content is negligible, then some arguments emerge [15] that the SP Regge trajectory tends to the unity in the limit of asymptotically high transferred momenta:

$\lim _{t \rightarrow-\infty} \alpha_{\mathrm{SP}}(t)=1$.

The analogous limit for the FR Regge trajectory is [16]

$\lim _{t \rightarrow-\infty} \alpha_{\mathrm{FR}}(t)=0$.

Thus, at $t \rightarrow-\infty$, the SP (FR) can be treated as a vector (scalar) virtual object. Hence, according to the so-called quark counting rules $[17,18]$, the asymptotic behavior of the $\mathrm{SP}$ and FR couplings to proton is expected to be

$\lim _{t \rightarrow-\infty} g_{\mathrm{SP}}^{(p)}(t)=O\left(|t|^{-2}\right), \quad \lim _{t \rightarrow-\infty} g_{\mathrm{FR}}^{(p)}(t)=O\left(|t|^{-3 / 2}\right)$. 


\subsection{Test parametrizations for the unknown functions}

Unfortunately, the above-mentioned restrictions on the $t$ behavior of the Regge trajectories and the Reggeon couplings to proton do not allow to determine unambiguously their analytic form. Therefore, in order to apply the proposed model to available experimental data, we have to introduce some test parametrizations. The simplest functions which satisfy the relations (21), (22), and (25)-(28) are the following ones:

$$
\begin{gathered}
\alpha_{\mathrm{SP}}(t)=1+\frac{\alpha_{\mathrm{SP}}(0)-1}{1-\frac{t}{\tau_{\mathrm{SP}}}}, \quad g_{\mathrm{SP}}^{(p)}(t)=\frac{g_{\mathrm{SP}}^{(p)}(0)}{\left(1-a_{1} t\right)^{2}}, \\
\alpha_{\mathrm{FR}}(t)=\frac{\alpha_{\mathrm{FR}}(0)}{1-\frac{t}{\tau_{\mathrm{FR}}}}, \quad g_{\mathrm{FR}}^{(p)}(t)=\frac{g_{\mathrm{FR}}^{(p)}(0)}{\left(1-a_{2} t\right)^{3 / 2}} .
\end{gathered}
$$

These parametrizations should be considered just as some rough phenomenological approximations to $\alpha_{\mathrm{SP}}(t), g_{\mathrm{SP}}^{(p)}(t)$, $\alpha_{\mathrm{FR}}(t)$, and $g_{\mathrm{FR}}^{(p)}(t)$ in the region of negative $t$ only. The true Regge trajectories and Reggeon couplings to proton have much more complicated $t$-behavior with branching points, and, certainly, they have no poles on the physical sheet. Therefore, the approximations (29) are absolutely invalid in the region of positive values of $t$, because the behavior $\alpha(t) \rightarrow \infty$ at finite $t$-values is impossible for true Regge trajectories.

Having substituted the functions (29) into (16), we obtain the expressions for the corresponding Reggeon contributions to the eikonal. Then, with the help of the approximation (20) and the eikonal representation (1) of the elastic scattering amplitude, we can treat the differential cross-section,

$\frac{d \sigma_{e l}}{d t}=\frac{\left|T_{N}(s, t)\right|^{2}}{16 \pi s^{2}}$,

as a function of $s$ and $t$ dependent, as well, on the model free parameters.

The next step is the fitting of the free parameter values to available data on the proton-proton EDS in the relevant kinematic range.

\section{Applying the model to experimental data on the nucleon-nucleon EDS angular distributions}

As we intend not only to describe available data, but, also, to check the model predictive value, so we should, first, fit the model parameter values to the limited dataset in the restricted kinematic range and, second, compare the model predictions for the differential cross-sections beyond this range with those data which were not included into the fitting procedure. For this purpose, the following set of data on the proton-proton EDS is chosen: the ISR data $[19,20]$ in the range $\{30 \mathrm{GeV}<\sqrt{s}<63 \mathrm{GeV}, 0.1 \mathrm{GeV} \leq \sqrt{-t} \leq$ $1.5 \mathrm{GeV}\}$, the high precision data at $\sqrt{s}=9.8 \mathrm{GeV}$ and
Table 1 The parameter values for (29) obtained via fitting to the limited dataset in the kinematic range $\{9.8 \mathrm{GeV} \leq \sqrt{s} \leq 200 \mathrm{GeV}, 0.1 \mathrm{GeV} \leq$ $\sqrt{-t} \leq 1.5 \mathrm{GeV}\}$

\begin{tabular}{ll}
\hline Parameter & Value \\
\hline$\alpha_{\mathrm{SP}}(0)-1$ & 0.114 \\
$\tau_{\mathrm{SP}}$ & $0.552 \mathrm{GeV}^{2}$ \\
$g_{\mathrm{SP}}^{(p)}(0)$ & $13.1 \mathrm{GeV}$ \\
$a_{1}$ & $0.276 \mathrm{GeV}^{-2}$ \\
$\alpha_{\mathrm{FR}}(0)$ & 0.61 \\
$\tau_{\mathrm{FR}}$ & $1.54 \mathrm{GeV}^{2}$ \\
$g_{\mathrm{FR}}^{(p)}(0)$ & $18.2 \mathrm{GeV}^{-2}$ \\
$a_{2}$ & $0.47 \mathrm{GeV}^{-2}$ \\
\hline
\end{tabular}

$0.01 \mathrm{GeV}^{2} \leq-t \leq 0.75 \mathrm{GeV}^{2}$ gathered at U-70 [21] and the Fermilab accelerator [22], and the data at $\sqrt{s}=200 \mathrm{GeV}$ and $0.045 \mathrm{GeV}^{2} \leq-t \leq 0.135 \mathrm{GeV}^{2}$ recently published by STAR Collaboration [13].

The fitting results are presented in Tables 1 and 2 and in Fig. 3. As well, Fig. 3 contains the ISR data in the range $\left\{30 \mathrm{GeV}<\sqrt{s}<63 \mathrm{GeV}, 0.05 \mathrm{GeV}^{2} \leq-t \leq 0.85 \mathrm{GeV}^{2}\right\}$ [23], the data at $\sqrt{s}=23.4 \mathrm{GeV}[19,20]$, and the data at lower energies taken from the collection [24]. We emphasize once again that only those data were included into the fitting procedure which were mentioned in Table 2 . The model curves outside of the kinematic ranges pointed out in this table should be considered as the predictions.

The predictions for the nucleon-nucleon differential cross-sections at ultrahigh energies [25-37] and for the $\bar{p} p$ angular distributions at $\sqrt{s} \leq 63 \mathrm{GeV}[19,23,24]$ are presented in Figs. 4 (solid lines) and 5, correspondingly. The quality of these predictions for the EDS data at $\sqrt{s}>20 \mathrm{GeV}$ is given in Tables 3 and 4 .

The model predictions for the $p p$ total cross-section and for $\rho=\frac{\operatorname{Re} T_{N}(s, 0)}{\operatorname{Im} T_{N}(s, 0)}$ at $\sqrt{s}=13 \mathrm{TeV}$ are $\sigma_{\text {tot }}^{\text {model }}(13 \mathrm{TeV})$ $\approx 109.6 \mathrm{mb}$ and $\rho^{\text {model }}(13 \mathrm{TeV}) \approx 0.129$, while the corresponding measured values are $\sigma_{\text {tot }}(13 \mathrm{TeV})=(110.5 \pm 2.4)$ $\mathrm{mb}$ and $\rho(13 \mathrm{TeV})=0.10 \pm 0.01$ [35]. It should be noted here that the extraction of these quantities (in particular, of the $\rho$-parameter) from the experimental angular distributions is a strongly model-dependent procedure.

Although the model demonstrates an impressive predictive value in the region of the SPS and Tevatron energies, one can observe significant deviations of the model curves from the data in the diffraction dip region at $\sqrt{s}<30 \mathrm{GeV}$ and $\sqrt{s} \geq 7 \mathrm{TeV}$ and, also, in the region of relatively high transferred momenta, $\sqrt{-t}>1.3 \mathrm{GeV}$. Let us explain these discrepancies. 
Table 2 The quality of the model description of the proton-proton EDS angular distributions

\begin{tabular}{|c|c|c|c|c|}
\hline References & $\sqrt{s}, \mathrm{GeV}$ & $t$-interval & Number of points & $\chi^{2}$ \\
\hline$[21]$ & 9.8 & $0.0124 \mathrm{GeV}^{2} \leq-t \leq 0.12 \mathrm{GeV}^{2}$ & 14 & 42 \\
\hline$[22]$ & 9.8 & $0.0375 \mathrm{GeV}^{2} \leq-t \leq 0.75 \mathrm{GeV}^{2}$ & 16 & 38 \\
\hline [19] & 30.7 & $0.01 \mathrm{GeV}^{2}<-t<0.018 \mathrm{GeV}^{2}$ & 10 & 3 \\
\hline$[20]$ & 30.5 & $0.875 \mathrm{GeV}^{2} \leq-t \leq 2.25 \mathrm{GeV}^{2}$ & 26 & 136 \\
\hline$[20]$ & 44.6 & $0.875 \mathrm{GeV}^{2} \leq-t \leq 2.25 \mathrm{GeV}^{2}$ & 26 & 105 \\
\hline [19] & 52.8 & $0.01 \mathrm{GeV}^{2}<-t<0.056 \mathrm{GeV}^{2}$ & 23 & 57 \\
\hline$[20]$ & 52.8 & $0.825 \mathrm{GeV}^{2} \leq-t \leq 2.25 \mathrm{GeV}^{2}$ & 27 & 157 \\
\hline [19] & 62.3 & $0.01 \mathrm{GeV}^{2}<-t<0.052 \mathrm{GeV}^{2}$ & 18 & 33 \\
\hline$[20]$ & 62.1 & $0.825 \mathrm{GeV}^{2} \leq-t \leq 2.25 \mathrm{GeV}^{2}$ & 27 & 167 \\
\hline$[13]$ & 200.0 & $0.045 \mathrm{GeV}^{2} \leq-t \leq 0.135 \mathrm{GeV}^{2}$ & 35 & 120 \\
\hline Total & & & 222 & 858 \\
\hline
\end{tabular}
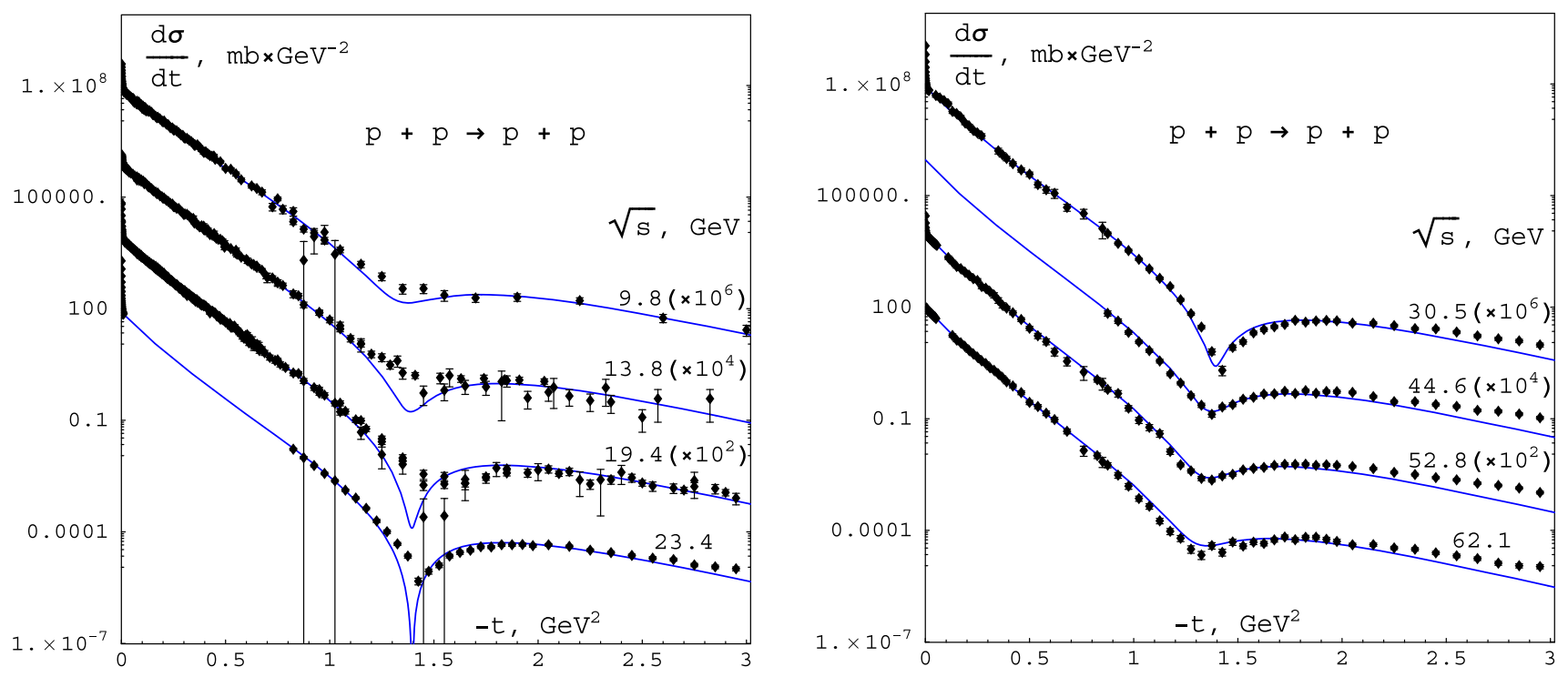

Fig. 3 The differential cross-sections of proton-proton EDS at high energies and low transferred momenta

\section{Discussion of the issues}

\subsection{Impact of other supercritical Reggeon contributions}

First of all, one can pay attention to the fact that the free parameter values related to the SP contribution are close to those obtained in the framework of the one-Reggeon approximation via fitting to the data on nucleon-nucleon EDS in the energy interval $546 \mathrm{GeV} \leq \sqrt{s} \leq 7 \mathrm{TeV}$ [38]. Hence, the divergence between the model curves and the LHC data can be explained in the same way as it was done in [39]. Namely, the reason is our ignoring of the contribution into the eikonal by the hard Pomeron (HP), also known as the BFKL Pomeron. This supercritical Reggeon dominates in the deeply inelastic scattering (DIS) of leptons on protons. The intercept of its Regge trajectory can be extracted from the data on the proton unpolarized structure function $F_{2}^{p}\left(x, Q^{2}\right)$
[40] at high values of the incoming photon virtuality $Q^{2}$ and low values of the Bjorken scaling variable $x: \alpha_{\mathrm{HP}}(0) \approx 1.32$ [41]. In spite of such a high value of $\alpha_{\mathrm{HP}}(0)$, the corresponding term in the eikonal is subdominant relative to the SP contribution up to the LHC energies, due to the extremely weak $t$-dependence of $\alpha_{\mathrm{HP}}(t)$ in the transferred momentum range relevant for EDS (a detailed discussion of this matter can be found in [42]).

If we add an extra term into the eikonal,

$\Omega_{\mathrm{HP}}(s, t)=\xi_{+}\left(\alpha_{\mathrm{HP}}(0)\right) \beta_{\mathrm{HP}}^{(p)}(t)\left(\frac{s}{2 s_{0}}\right)^{\alpha_{\mathrm{HP}}(0)}$,

where

$\beta_{\mathrm{HP}}^{(p)}(t) \equiv g_{\mathrm{HP}}^{(p) 2}(t) \alpha_{\mathrm{HP}}^{\prime}(t)=\beta_{\mathrm{HP}}^{(p)}(0) e^{b t}$ 

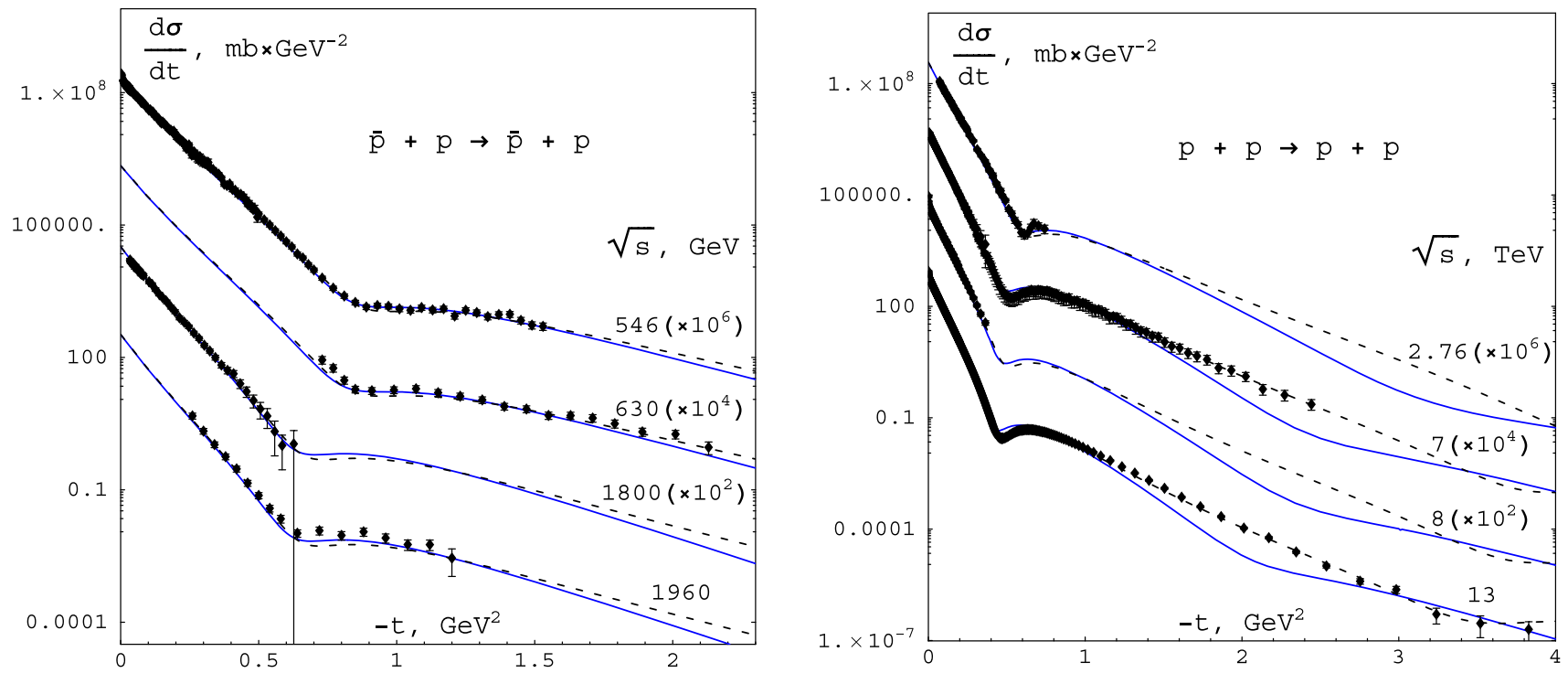

Fig. 4 The differential cross-sections of nucleon-nucleon EDS at ultrahigh energies. The solid lines correspond to the two-Reggeon eikonal model. The dashed lines are related to the three-Reggeon approximation wherein the HP exchange contribution is taken into account

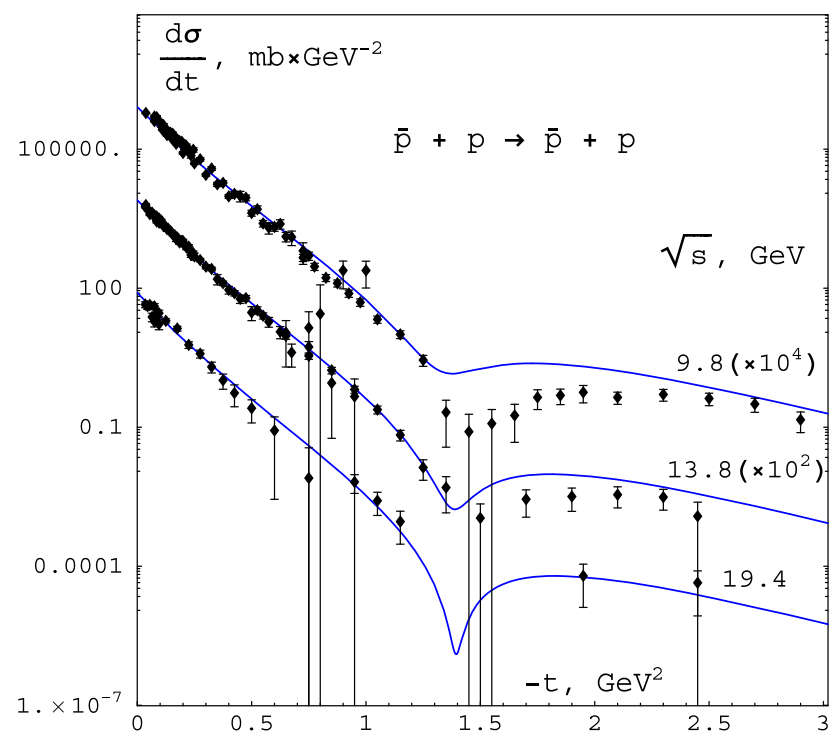

Fig. 5 The $\bar{p} p$ EDS angular distributions

is the HP Regge residue, $\beta_{\mathrm{HP}}^{(p)}(0)=0.0335$ and $b=$ $1.6 \mathrm{GeV}^{-2}$, then the deviations of the model curves from the LHC data become noticeably weaker (see Fig. 4 (dashed lines) and Table 3 ).

However, the HP influence is crucial only if we consider the angular distributions in the dip region and at transferred momenta higher than $1 \mathrm{GeV}$. The HP exchanges have a negligible impact on the integrated cross-section values $[12,13,43]$ (Fig. 6) and on the differential cross-section curves in the Coulomb-nuclear interference (CNI) region [33,35] (Fig. 7). Here we take account of the electromagnetic interaction via direct adding of the Coulomb term to the full amplitude related to strong interaction:

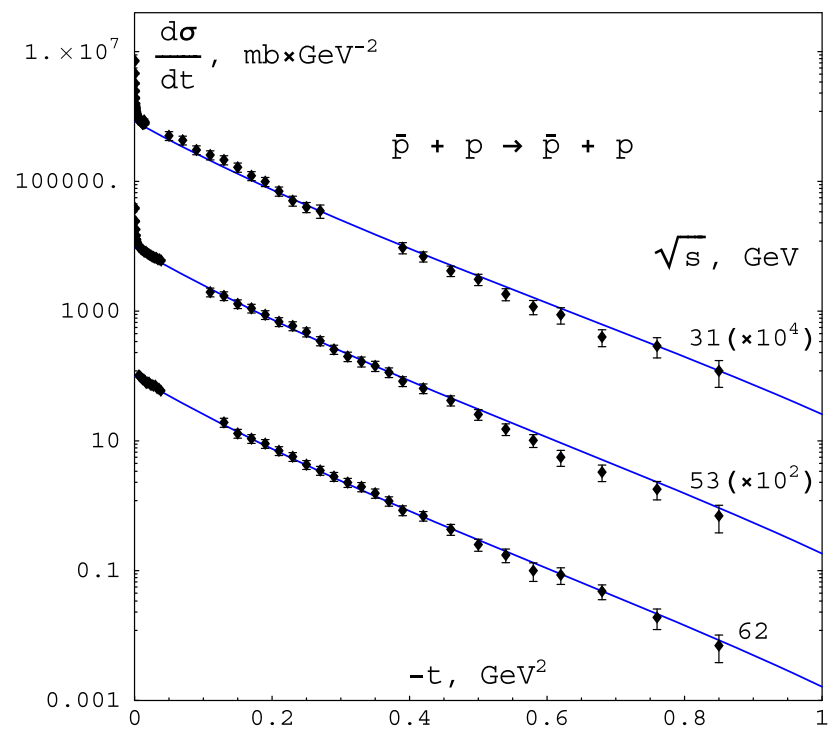

$$
\begin{gathered}
T_{N}(s, t) \rightarrow \Omega_{C}(s, t)+T_{N}(s, t), \\
\Omega_{C}(s, t) \approx \mp \frac{8 \pi s \alpha_{e}}{t} F_{E}^{2}(t),
\end{gathered}
$$

where $F_{E}(t)=\left(1-\frac{t}{0.71 \mathrm{GeV}^{2}}\right)^{-2}$ is the dipole electric form-factor of proton, and $\alpha_{e}$ is the fine structure constant. ${ }^{2}$

\footnotetext{
$\overline{2}$ It was argued in [39] that, in the leading approximation, the CNI can be ignored in the amplitude level. As well, it was demonstrated in [44] on an example of two independent models that, at the LHC energies, such an approximation leads to a small (not higher than 3.5\%) overestimation of the differential cross-section. A more detailed discussion of the CNI phenomenon can be found in [45].
} 
Table 3 The quality of the model predictions for the nucleon-nucleon EDS angular distributions at ultrahigh energies. The numbers in parentheses are related to the three-Reggeon eikonal approximation wherein the HP exchange contribution is taken into account

\begin{tabular}{|c|c|c|c|c|}
\hline Experiment & $\sqrt{s}, \mathrm{TeV}$ & $t$-interval & Number of points & $\chi^{2}$ \\
\hline $\mathrm{UA} 4(\bar{p} p)$ & 0.546 & $0.01 \mathrm{GeV}^{2}<-t<0.035 \mathrm{GeV}^{2}$ & 50 & $43(50)$ \\
\hline $\mathrm{UA} 4(\bar{p} p)$ & 0.546 & $0.03 \mathrm{GeV}^{2}<-t<0.5 \mathrm{GeV}^{2}$ & 87 & $224(143)$ \\
\hline $\mathrm{UA4}(\bar{p} p)$ & 0.546 & $0.46 \mathrm{GeV}^{2} \leq-t \leq 1.53 \mathrm{GeV}^{2}$ & 34 & $36(25)$ \\
\hline $\mathrm{UA} 4(\bar{p} p)$ & 0.63 & $0.73 \mathrm{GeV}^{2} \leq-t \leq 2.13 \mathrm{GeV}^{2}$ & 19 & $31(27)$ \\
\hline $\mathrm{E} 710(\bar{p} p)$ & 1.8 & $0.03 \mathrm{GeV}^{2}<-t<0.63 \mathrm{GeV}^{2}$ & 51 & $20(17)$ \\
\hline $\mathrm{D} 0(\bar{p} p)$ & 1.96 & $0.26 \mathrm{GeV}^{2} \leq-t \leq 1.2 \mathrm{GeV}^{2}$ & 17 & $31(28)$ \\
\hline TOTEM $(p p)$ & 2.76 & $0.07 \mathrm{GeV}^{2}<-t<0.75 \mathrm{GeV}^{2}$ & 63 & $455(268)$ \\
\hline TOTEM $(p p)$ & 7.0 & $0.01 \mathrm{GeV}^{2}<-t<2.5 \mathrm{GeV}^{2}$ & 161 & $206(115)$ \\
\hline ATLAS $(p p)$ & 7.0 & $0.01 \mathrm{GeV}^{2}<-t<0.4 \mathrm{GeV}^{2}$ & 39 & $53(52)$ \\
\hline TOTEM $(p p)$ & 8.0 & $0.01 \mathrm{GeV}^{2}<-t<0.2 \mathrm{GeV}^{2}$ & 22 & $143(75)$ \\
\hline $\operatorname{ATLAS}(p p)$ & 8.0 & $0.01 \mathrm{GeV}^{2}<-t<0.4 \mathrm{GeV}^{2}$ & 39 & $145(193)$ \\
\hline TOTEM $(p p)$ & 13.0 & $0.01 \mathrm{GeV}^{2}<-t<3.9 \mathrm{GeV}^{2}$ & 403 & $9123(2566)$ \\
\hline
\end{tabular}

Table 4 The quality of the model description of those ISR data on nucleon-nucleon EDS which were not included into the fitting procedure

\begin{tabular}{|c|c|c|c|c|}
\hline References & $\sqrt{s}, \mathrm{GeV}$ & $t$-interval & Number of points & $\chi^{2}$ \\
\hline [20] & $23.4(p p)$ & $0.825 \mathrm{GeV}^{2} \leq-t \leq 2.25 \mathrm{GeV}^{2}$ & 27 & 217 \\
\hline [23] & $31.0(p p)$ & $0.05 \mathrm{GeV}^{2} \leq-t \leq 0.85 \mathrm{GeV}^{2}$ & 24 & 28 \\
\hline [23] & $53.0(p p)$ & $0.11 \mathrm{GeV}^{2} \leq-t \leq 0.85 \mathrm{GeV}^{2}$ & 24 & 21 \\
\hline [23] & $62.0(p p)$ & $0.13 \mathrm{GeV}^{2} \leq-t \leq 0.85 \mathrm{GeV}^{2}$ & 23 & 14 \\
\hline [19] & $30.7(\bar{p} p)$ & $0.01 \mathrm{GeV}^{2}<-t<0.016 \mathrm{GeV}^{2}$ & 8 & 54 \\
\hline [23] & $31.0(\bar{p} p)$ & $0.05 \mathrm{GeV}^{2} \leq-t \leq 0.85 \mathrm{GeV}^{2}$ & 22 & 21 \\
\hline [19] & $52.8(\bar{p} p)$ & $0.01 \mathrm{GeV}^{2}<-t<0.039 \mathrm{GeV}^{2}$ & 16 & 70 \\
\hline [23] & $53.0(\bar{p} p)$ & $0.11 \mathrm{GeV}^{2} \leq-t \leq 0.85 \mathrm{GeV}^{2}$ & 24 & 21 \\
\hline [19] & $62.3(\bar{p} p)$ & $0.01 \mathrm{GeV}^{2}<-t<0.039 \mathrm{GeV}^{2}$ & 14 & 40 \\
\hline [23] & $62.0(\bar{p} p)$ & $0.13 \mathrm{GeV}^{2} \leq-t \leq 0.85 \mathrm{GeV}^{2}$ & 23 & 5 \\
\hline
\end{tabular}

Our ignoring of the HP exchanges in the framework of the two-Reggeon model is not the only reason for the model curve deviations from the data at the LHC energies. Some extra supercritical Reggeons should be included into consideration for the a better description, namely, the soft and hard Odderons (the $C$-odd counterpartners of the SP and HP, correspondingly). The exchanges by these Reggeons lead to a visible splitting of the $\bar{p} p$ and $p p$ angular distributions in the diffraction dip region [46]. Nonetheless, the Odderons' combined influence on the shape of the nucleon-nucleon EDS differential cross-section at ultrahigh energies seems to be a much finer effect than the impact of the HP exchanges, and, thus, it can be ignored, in the leading approximation.

\subsection{Impact of secondary Reggeons}

To understand how strong is the influence of secondary Reggeons on the considered differential cross-sections, let us explore the impact of the FR exchanges on the proton- proton EDS angular distribution at $\sqrt{s}=52.8 \mathrm{GeV}$. In Fig. 8 , two curves are compared. The first one is obtained in the framework of the proposed two-Reggeon approximation (the solid line). The second one is related to the one-Reggeon approximation wherein the FR contribution into the eikonal is ignored (the dashed line), while the functions $\alpha_{\mathrm{SP}}(t)$ and $g_{\mathrm{SP}}^{(p)}(t)$ are kept the same. One can see that the impact of the FR exchanges on the differential cross-section shape is the most significant in the region of transferred momenta higher than $1 \mathrm{GeV}$. The distortion of the diffraction pattern at lower values of the transferred momentum is not so crucial.

Consequently, the observed deviations of the model curves from the data on the $\bar{p} p$ EDS at $\sqrt{s}<15 \mathrm{GeV}$ (see the left picture in Fig. 5) can be explained by our ignoring of the combined influence of secondary Reggeons (namely, the $f, \omega, a$, and $\rho$ contributions of type 2 discussed above in Sect. 3.1). The impact of secondaries goes down fast with energy and becomes almost negligible in the region $\sqrt{s}>30 \mathrm{GeV}$. In its turn, the splitting between the $p p$ 


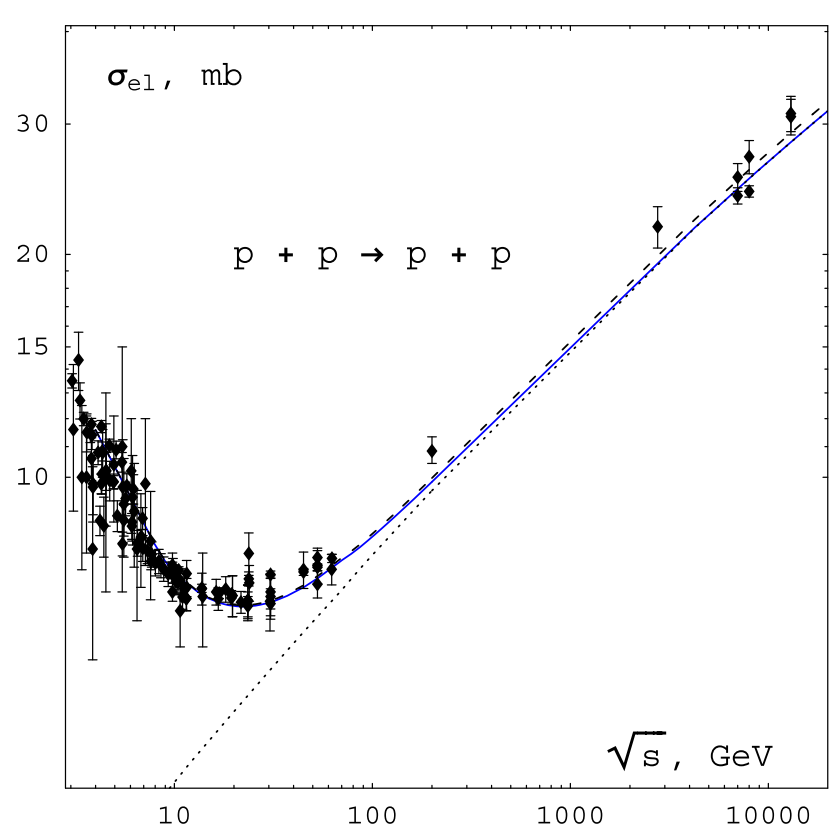

Fig. 6 The integrated cross-sections of nucleon-nucleon EDS. The solid lines correspond to the two-Reggeon eikonal model. The dashed lines are related to the three-Reggeon approximation, wherein the HP

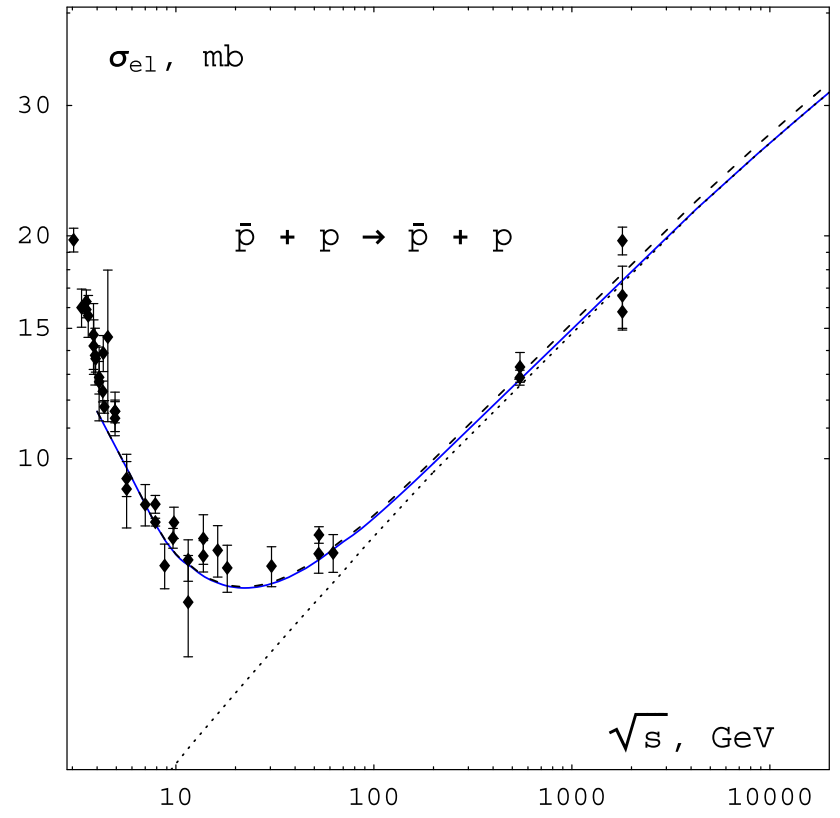

exchange contribution is taken into account. The dotted lines correspond to the one-Reggeon eikonal model, wherein just the SP exchanges are taken into account
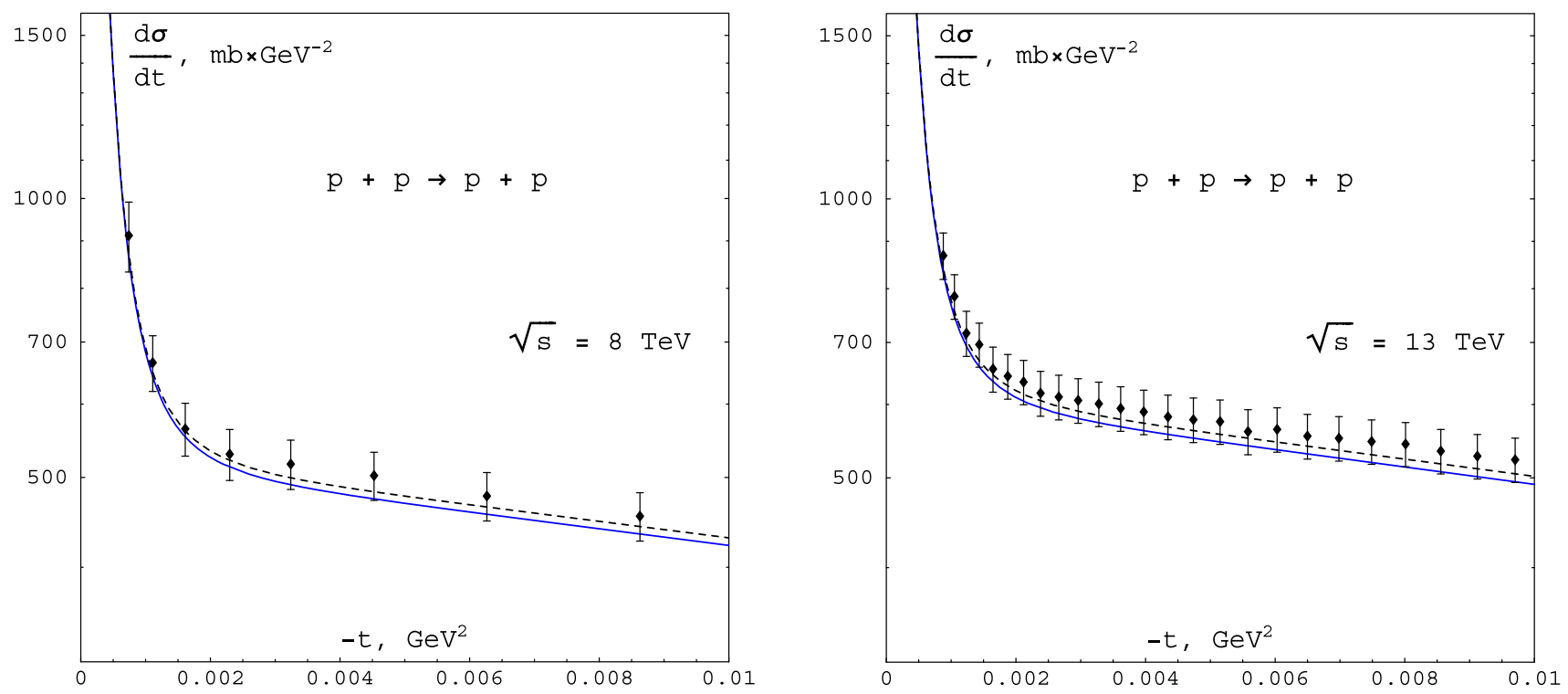

Fig. 7 The differential cross-sections of proton-proton EDS in the CNI region. The solid lines correspond to the two-Reggeon eikonal model. The dashed lines are related to the three-Reggeon approximation, wherein the HP exchange contribution is taken into account

and $\bar{p} p$ angular distributions at the ISR energies in the CNI region [19] is, mainly, owing to electromagnetic interaction (see Fig. 9). In other words, the combined contribution of secondary Reggeon exchanges into the eikonal is so subdominant that the two-Reggeon eikonal approximation turns out quite applicable for qualitative description of the $\bar{p} p$ EDS at $\sqrt{s}>30 \mathrm{GeV}$.
5.3 Other sources of divergences between the model curves and the experimental data

The spin phenomena in the high-energy EDS of nucleons are negligible only at small enough values of the transferred momentum. Certainly, they should not be ignored in the region where the condition (12) is invalid. Hence, the notice- 

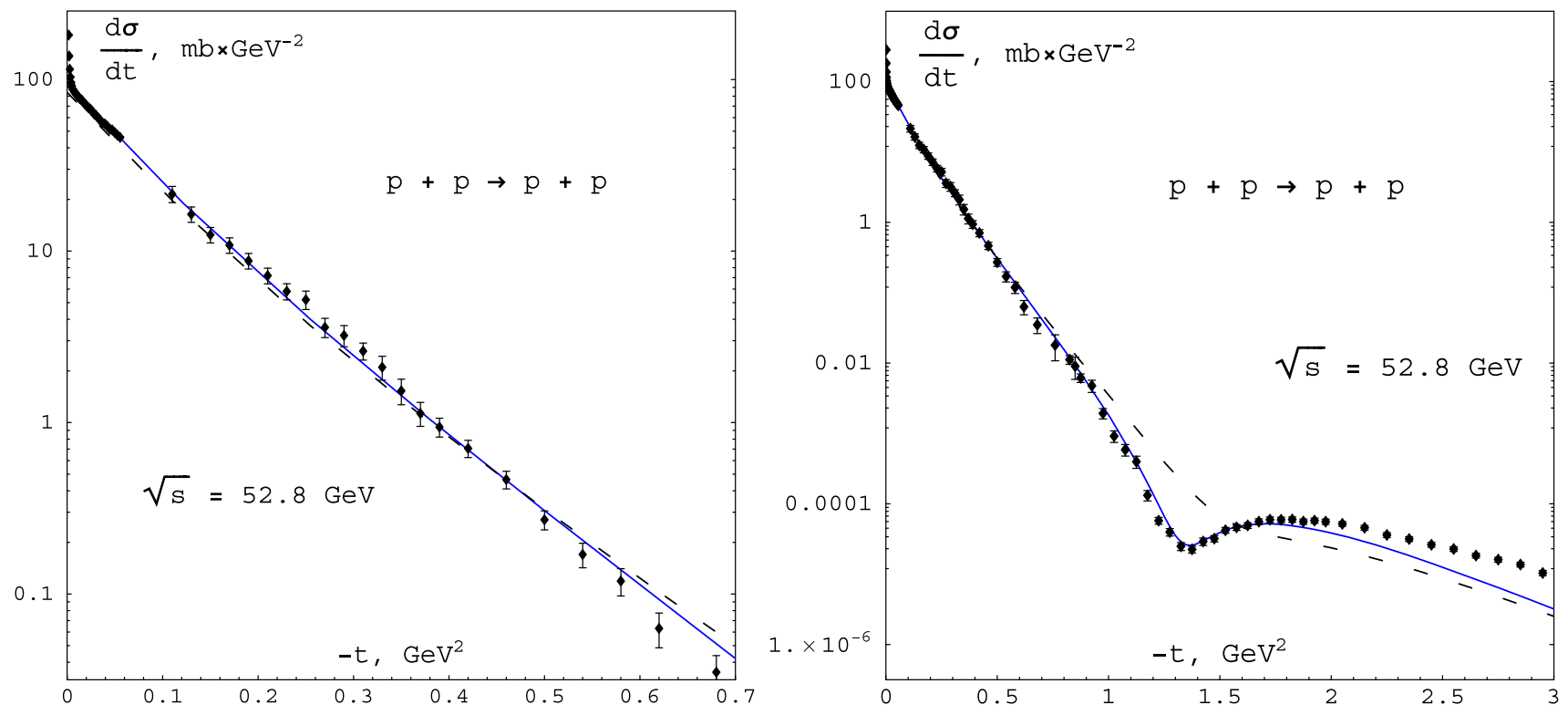

Fig. 8 The $p$ p EDS angular distribution at $\sqrt{s}=52.8 \mathrm{GeV}$. The solid lines correspond to the two-Reggeon eikonal model. The dashed lines are related to the one-Reggeon eikonal approximation wherein the FR exchange contribution to the eikonal is ignored
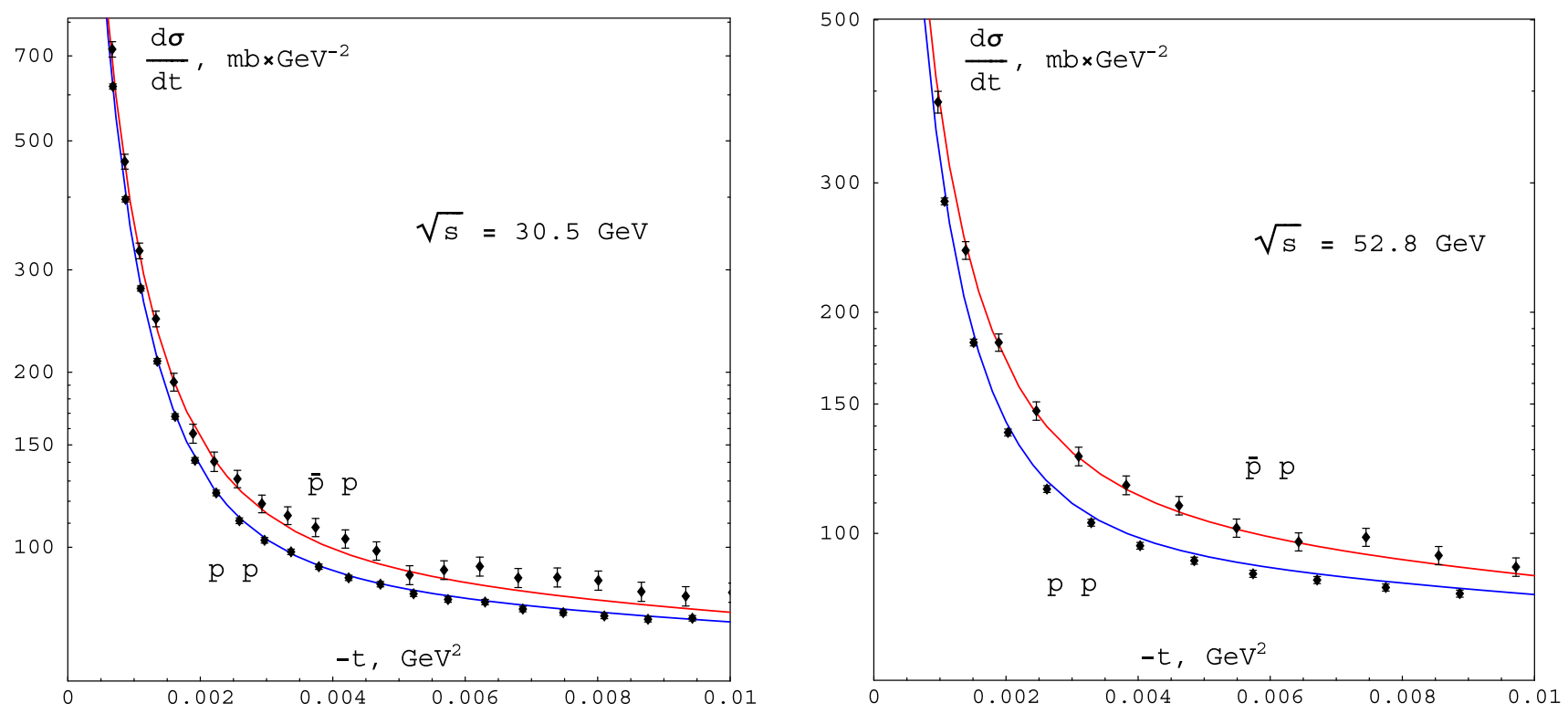

Fig. 9 The differential cross-sections of the $p p$ and $\bar{p} p$ EDS at the ISR energies in the CNI region

able underestimation of the proton-proton EDS differential cross-sections at $\sqrt{s}<63 \mathrm{GeV}$ and $\sqrt{-t}>1.3 \mathrm{GeV}$ (see Fig. 3) can be related (at least, in part) to spin-flip effects.

Another reason of this underestimation is our choice of parametrizations for $\alpha_{\mathrm{SP}}(t), g_{\mathrm{SP}}^{(p)}(t), \alpha_{\mathrm{FR}}(t)$, and $g_{\mathrm{FR}}^{(p)}(t)$. The true SP and FR Regge trajectories and couplings to proton have much more complicated analytic behavior than the functions presented in (29). For instance, $g_{\mathrm{SP}}^{(p)}(t)$ and $g_{\mathrm{FR}}^{(p)}(t)$ even may be nonmonotonic at high enough values of the transferred momentum. Nonetheless, we restrict ourselves by the simplest parametrizations satisfying the relations (21), (22), and (25)-(28) to make our reasoning more transparent and conclusions more justified. In other words, we have constructed the roughest model applicable for qualitative description of nucleon-nucleon EDS in a wide interval of the collision energy values.

However, the simplicity of the proposed two-Reggeon approximation is not the only feature which distinguishes it from other models exploiting the notion of Reggeons. 


\subsection{Distinction from other Reggeon models}

Modern models for high-energy elastic scattering of hadrons based on Regge theory can be divided into two groups: Regge-eikonal models [47-49] and those models which take account of single and double Reggeon exchanges but ignore higher order terms in the eikonal power expansion of the scattering amplitude [50-52].

The primary distinction of the proposed scheme from the above-mentioned models is the exploitation of nonlinear approximations to Regge trajectories in the region of negative values of the argument. A detailed discussion of why any hadron Regge trajectory must exhibit an essentially nonlinear behavior at $t<0$ and how such a nonlinearity may be consistent with the approximately linear behavior in the resonance region, $t>0$, can be found in [53]. Here we restrict ourselves just by one of the main arguments.

Usage of linear parametrizations for Regge trajectories leads to the emergence of simple poles in the real part of the Reggeon signature factor (17) at those negative values of $t$ where the corresponding Regge trajectory takes on integers $0,-2,-4, \ldots$ Various models resort to various tricks to avoid this issue. For instance, in [47], the functions $\alpha(t)$ in the signature factors are replaced by $\alpha(0)$. Other models, as well, exploit some expressions for the even Reggeon signature factor which are different from (17). However, that is not quite correct, because the Sommerfeld-Watson transform leads exactly to the formula (17) [2], and, hence, the expression (17) is the only correct form of this factor. As a result, despite of its roughness and stiffness, the considered approximation exhibits a much higher predictive value than other Reggeon models, concerning the energy evolution of the diffraction pattern of nucleon-nucleon elastic scattering.

Another feature of the proposed model is its abovediscussed simplicity in the aspect of both the Reggeon structure of the scattering amplitude and the parametrizations of the Regge trajectories and residues. The vast majority of other models have a much more complicated Reggeon and parametric structure. For instance, three Pomerons are introduced within the model published in [47], the relatively simple model considered in [50] includes the exchanges by the $\omega$-Reggeon with the trajectory significantly different from the Regge trajectory of the $f$-Reggeon, while the model proposed in [52] introduces the so-called Froissaron and Maximal Odderon in addition to the usual simple $j$-pole Pomeron and Odderon and exploits a much higher number of free parameters as compared to other models. More complicated phenomenological structure makes these models more flexible and allows to include into consideration the kinematic region of transferred momenta higher than $1.5 \mathrm{GeV}$ and, as well, to obtain a more satisfactory (from the statistical standpoint) description of available data, though the most of the above-mentioned models do not deal with the data in the collision energy region $\sqrt{s}<19 \mathrm{GeV}$.

\section{Conclusions}

In view of the aforesaid, we have to conclude that the considered two-Reggeon eikonal model is a simple and reliable phenomenological tool which provides a qualitative description of the $p p$ EDS in the kinematic range $\{10 \mathrm{GeV} \leq \sqrt{s} \leq$ $2 \mathrm{TeV}, \sqrt{-t}<1.5 \mathrm{GeV}\}$ and of the $\bar{p} p$ EDS in the kinematic range $\{30 \mathrm{GeV} \leq \sqrt{s} \leq 2 \mathrm{TeV}, \sqrt{-t}<1.5 \mathrm{GeV}\}$. The ultimate dominance of the SP and FR over other Reggeons in the EDS of nucleons up to the LHC energies is not just an artificial pattern but a real physical phenomenon. The obtained approximations to the SP and FR Regge trajectories and couplings to proton can be used in the framework of those Reggeon models which describe various reactions of high-energy inelastic diffractive scattering of nucleons, including single and double diffractive dissociation, central exclusive production of light vacuum resonance states, etc.

Data Availability Statement This manuscript has no associated data or the data will not be deposited. [Authors' comment: This paper is not experimental. All the sources of the used experimental data are properly cited in the list of References and referred in the text.]

Open Access This article is licensed under a Creative Commons Attribution 4.0 International License, which permits use, sharing, adaptation, distribution and reproduction in any medium or format, as long as you give appropriate credit to the original author(s) and the source, provide a link to the Creative Commons licence, and indicate if changes were made. The images or other third party material in this article are included in the article's Creative Commons licence, unless indicated otherwise in a credit line to the material. If material is not included in the article's Creative Commons licence and your intended use is not permitted by statutory regulation or exceeds the permitted use, you will need to obtain permission directly from the copyright holder. To view a copy of this licence, visit http://creativecomm ons.org/licenses/by/4.0/.

Funded by SCOAP ${ }^{3}$.

\section{References}

1. A.A. Godizov, arXiv:1203.6013v3 [hep-ph]

2. P.D.B. Collins, An Introduction to Regge Theory \& High Energy Physics (Cambridge University Press, Cambridge, 1977)

3. A.V. Kisselev, V.A. Petrov, Nuovo Cim. A 106, 1087 (1993)

4. L. Van Hove, Phys. Lett. B 24, 183 (1967)

5. STAR Collaboration, Phys. Lett. B 719, 62 (2013)

6. A. Gaidot et al., Phys. Lett. B 61, 103 (1976)

7. R.V. Kline et al., Phys. Rev. D 22, 553 (1980)

8. G. Fidecaro et al., Phys. Lett. B 105, 309 (1981)

9. O.V. Selyugin, Symmetry 13, 164 (2021)

10. G.N. Watson, Proc. R. Soc. 95, 83 (1918)

11. A. Sommerfeld, Partial Differential Equations in Physics (Academic Press, New York, 1949) 
12. Particle Data Group, https://pdg.lbl.gov/2021/hadronic-xsections/ rpp2021-pp_elastic.dat

13. STAR Collaboration, Phys. Lett. B 808, 135663 (2020)

14. G.C. Rossi, G. Veneziano, Nucl. Phys. B 123, 507 (1977)

15. P.D.B. Collins, P.J. Kearney, Z. Phys. C 22, 277 (1984)

16. J. Kwiecinski, Phys. Rev. D 26, 3293 (1982)

17. V.A. Matveev, R.M. Muradyan, A.N. Tavkhelidze, Lett. Nuovo Cim. 5, 907 (1972)

18. S.J. Brodsky, G.R. Farrar, Phys. Rev. Lett. 31, 1153 (1973)

19. N. Amos et al., Nucl. Phys. B 262, 689 (1985)

20. E. Nagy et al., Nucl. Phys. B 150, 221 (1979)

21. I.M. Geshkov et al., Phys. Rev. D 13, 1846 (1976)

22. D.S. Ayres et al., Phys. Rev. D 15, 3105 (1977)

23. A. Breakstone et al., Nucl. Phys. B 248, 253 (1984)

24. J.R. Cudell, A. Lengyel, E. Martynov, https://www.theo.phys.ulg. ac.be/ cudell/data

25. UA4 Collaboration, M. Bozzo et al., Phys. Lett. B 147, 385 (1984)

26. UA4 Collaboration, M. Bozzo et al., Phys. Lett. B 155, 197 (1985)

27. UA4 Collaboration, D. Bernard et al., Phys. Lett. B 198, 583 (1987)

28. UA4 Collaboration, D. Bernard et al., Phys. Lett. B 171, 142 (1986)

29. E-710 Collaboration, N. Amos et al., Phys. Lett. B 247, 127 (1990)

30. D0 Collaboration, Phys. Rev. D 86, 012009 (2012)

31. TOTEM Collaboration, Europhys. Lett. 101, 21002 (2013)

32. ATLAS Collaboration, Nucl. Phys. B 889, 486 (2014)

33. TOTEM Collaboration, Eur. Phys. J. C 76, 661 (2016)

34. ATLAS Collaboration, Phys. Lett. B 761, 158 (2016)

35. TOTEM Collaboration, Eur. Phys. J. C 79, 785 (2019)
36. TOTEM Collaboration, Eur. Phys. J. C 79, 861 (2019)

37. TOTEM Collaboration, Eur. Phys. J. C 80, 91 (2020)

38. A.A. Godizov, Eur. Phys. J. C 75, 224 (2015)

39. A.A. Godizov, Phys. Rev. D 101, 074028 (2020)

40. The H1 and ZEUS Collaborations, JHEP 1001, 109 (2010). http:// www.desy.de/h1zeus/combined_results

41. A.A. Godizov, Nucl. Phys. A 927, 36 (2014)

42. A.A. Godizov, Phys. Rev. D 96, 034023 (2017)

43. Particle Data Group, https://pdg.lbl.gov/2021/hadronic-xsections/ rpp2021-pbarp_elastic.dat

44. J. Kašpar, Acta Phys. Pol. B 52, 85 (2021)

45. V.A. Petrov, Eur. Phys. J. C 78, 221 (2018)

46. V.M. Abazov et al. (D0 Collaboration, TOTEM Collaboration), Phys. Rev. Lett. 127, 062003 (2021)

47. V.A. Petrov, A. Prokudin, Phys. Rev. D 87, 036003 (2013)

48. O.V. Selyugin, Nucl. Phys. A 959, 116 (2017)

49. V.A. Khoze, A.D. Martin, M.G. Ryskin, Phys. Lett. B 784, 192 (2018)

50. A. Donnachie, P.V. Landshoff, Phys. Lett. B 727, 500 (2013)

51. Particle Data Group, K.A. Olive et al., Chin. Phys. C 38, 090001 (2014) (page 541)

52. E. Martynov, B. Nicolescu, Eur. Phys. J. C 79, 461 (2019)

53. A.A. Godizov, V.A. Petrov, JHEP 0707, 083 (2007) 